\title{
Prevention of hypermelanosis by rearing Japanese flounder Paralichthys olivaceus in net-lined tanks
}

\author{
Koei Mizutani ${ }^{1,5} \cdot$ Toshiyuki Yamada $^{2} \cdot$ Keita W. Suzuki $^{3} \cdot$ Reiji Masuda $^{3} \cdot$ Kuniaki Nakata $^{4,6} \cdot$ Masatomo Tagawa $^{4}(\mathbb{0}$
}

Received: 6 July 2019 / Accepted: 26 September 2019

(c) The Author(s) 2019, corrected publication 2021

\begin{abstract}
In artificially reared flatfish, especially the Japanese flounder Paralichthys olivaceus, pigmented skin (hypermelanosis) frequently appears on the fish's blind side after normal metamorphosis. As no practical prevention method has yet been proposed, we examined the effectiveness of a loose net placed inside the rearing tank that covers the bottom and walls like a pouch. When juveniles (standard length [SL] $6 \mathrm{~cm}$ ) were transferred to the net-lined tank (mesh size $4 \mathrm{~mm}$ ) before the first appearance of hypermelanosis, the pigmented area after 2 months covered about $0.5 \%$ of the blind side; this is about $1 / 40$ th of the area covered by pigment in fish reared in an ordinary tank (20\%). Although the initial appearance of pigmentation in the axilla area (the area covered by the pectoral fin) was not suppressed, utilization of a larger mesh size (12 $\mathrm{mm})$ decreased the expansion of pigmentation in this area. Juveniles reared in the net-lined tank were about 5-15\% smaller (SL) than those reared in the ordinary tank, but their body depth:SL ratio was closer to that of wild-caught juveniles. From the results of this study, we propose that net-lined rearing tanks with larger-sized mesh are a practical method of preventing hypermelanosis in Japanese flounder aquaculture systems.
\end{abstract}

Keywords Abnormal skin color $\cdot$ Net-lined rearing tank $\cdot$ Flatfish aquaculture $\cdot$ Pleuronectiformes $\cdot$ Axilla darkening $\cdot$ Larger mesh size

\section{Introduction}

Most teleosts undergo striking bodily changes during their development from larva to juvenile (e.g., metamorphosis). The Japanese flounder Paralichthys olivaceus, a member

Masatomo Tagawa

tagawa@kais.kyoto-u.ac.jp

Koei Mizutani

k-mizutani31@pref.kyoto.lg.jp

Toshiyuki Yamada

yamadat@pref.nagasaki.lg.jp

Keita W. Suzuki

suzuki.keita.3r@kyoto-u.ac.jp

Reiji Masuda

reiji@kais.kyoto-u.ac.jp

Kuniaki Nakata

nakata@iwatani.co.jp

1 Department of Bioresource Science, Faculty of Agriculture,

Kyoto University, Kitashirakawa, Sakyo, Kyoto 606-8502,

Japan of order Pleuronectiformes, has, like other flatfish, symmetrically located eyes in its larval form. During metamorphosis, the right eye relocates to the left side of the body; thus, the left and right sides of the body are referred to as the ocular and blind sides, respectively. Adult-type

2 Nagasaki Prefectural Institute of Fisheries, Taira, Nagasaki, Nagasaki 851-2213, Japan

3 Maizuru Fisheries Research Station, Field Science Education and Research Center, Kyoto University, Nagahama, Maizuru, Kyoto 625-0086, Japan

4 Division of Applied Biosciences, Graduate School of Agriculture, Kyoto University, Kitashirakawa, Sakyo, Kyoto, Kyoto 606-8502, Japan

5 Present Address: Fisheries Office of Kyoto Prefecture, Odashukuno, Miyazu, Kyoto 626-0052, Japan

6 Present Address: Iwatani Corporation, Hommachi 3-6-4, Chuo-ku, Osaka, Osaka 541-0053, Japan 
melanophores and xanthophores appear on the skin of the ocular side, which becomes dark brown, but these are absent from the skin of the blind side, which remains white (Seikai et al. 1987; Nakamura et al. 2010).

Extensive seed production for aquaculture and stock enhancement has been carried out on the Japanese flounder due to its economic importance for Japanese fisheries. However, various color abnormalities occur during rearing in hatcheries, with one of the most significant of these being hypermelanosis. In hypermelanosis, first localized pigmented/darkened areas appear on the skin, followed by gradual expansion of the pigmented areas on the blind side of the flounder after the completion of normal metamorphosis. The market value of flounders with hypermelanosis, both those reared to harvest size in aquaculture systems and those released into the sea for recapture by fishermen, is about 20-70\% lower than that of fish with normal coloration (Kaji and Fukunaga 1999). Consequently, many studies have focused on strategies to prevent hypermelanosis in the Japanese flounder. Light-colored tank bottoms have been found to decrease or delay hypermelanosis (Yamanome et al. 2005; Amiya et al. 2005; Kang and Kim 2013b), an observation that has lead investigators to assess the contributions of melanophore-related hormones. Melanophore-stimulating hormone and its precursor have been investigated as possible stimulators (Yamanome et al. 2007b; Kang and Kim 2012, 2015), and melanin-concentrating hormone as a possible suppressor (Yamanome et al. 2005, 2007a; Kang and Kim 2012, 2013a). Gene expression studies of these hormones' receptors has provided evidence supporting their contribution to hypermelanosis (Matsuda et al. 2018a). Stress has also been studied, and a higher occurrence of hypermelanosis has been empirically associated with stressful conditions, with cortisol, a stress-responsive hormone, found to enhance hypermelanosis (Matsuda et al. 2018b). In addition, dietary studies suggest that vitamins A (Tarui et al. 2006) and D (Haga et al. 2004) have a stimulatory effect on hypermelanosis. However, despite these extensive and intensive efforts, hypermelanosis has proven difficult to prevent on the industrial scale.

The effect of simulating the ocean environment with a sandy substrate has been examined by several groups independently, with all studies showing a consistent and strong suppression of hypermelanosis (Seikai 1991; Iwata and Kikuchi 1998; Kang and Kim 2012, 2013a, b, c; Isojima et al. 2013b, 2014). However, this method is not employed in large-scale tanks because the sandy bottom interferes with satisfactory cleaning of the tank, particularly in terms of washing out residual food and excrement. The results of a previous study by our group, designed to find a sand substitute for juvenile Japanese flounders that already expressed a small degree of hypermelanosis, suggested that undulated tank bottoms were effective in suppressing hypermelanosis expansion (Nakata et al. 2017). By examining the pattern of contact between the blind side skin and the bottom surface of the tank, we proposed that contact stimulus from the bottom exerted a suppressive effect on hypermelanosis in the contact area.

We then tested the effect of a loosely set net that lined the inner surface of the rearing tank as an easily installable undulated bottom. The results of that study suggested the effectiveness of this net lining in preventing the expansion of hypermelanosis (Nakata et al. 2017). However, we did not test the effectiveness of the net lining in preventing the initial appearance of hypermelanosis. Therefore, the main purpose of the study reported here was to examine the suppressive effects of a net-lined rearing tank on the initial appearance and subsequent expansion of hypermelanosis in juvenile flounders, with the experiments starting before the appearance of any darkened areas. In addition, because the most difficult area in which to suppress darkening is the base of the pectoral fin (axilla area), additional experiments were conducted to clarify the reason for this local specificity. The applicability of net-lined rearing tanks for industrial use is discussed in relation to growth and body proportion, and directions for further research are suggested.

\section{Material and methods}

\section{Experiment 1: Effect of a net-lined rearing tank on juveniles before the appearance of hypermelanosis}

This experiment was carried out at the Nagasaki Prefectural Institute of Fisheries. Juvenile Japanese flounders, hatched from eggs spawned by artificially-grown adults, were kindly provided by a private hatchery (Ootawa Shubyo, Saikai, Nagasaki, Japan).

For the rearing experiment, two circular tanks (1000 1, transparent polycarbonate) were used, one for control rearing (control tank) and one for net-lined rearing (net tank). In the control tank, the juveniles were reared directly in the tank. In the net tank, netting (wind-protection net, polyethylene, blue, Raschel knitted, mesh size $4 \mathrm{~mm}$; Dio Chemicals Ltd, Tokyo, Japan) was fashioned into a large pouch of about the same size as the tank and loosely installed inside the tank (Fig. 1a). The juveniles were then reared inside the net pouch. Because the net pouch was simply inserted into the tank and only fixed along the surface, the net fabric undulated randomly. To ensure that the color and pattern of the bottom in the net tank and control tank were the same, we laid the same type of netting used in the lining under the transparent bottom of the control tank and placed both tanks on a white styrofoam sheet. Uniform color conditions were obtained by covering the outside of the vertical walls of both 


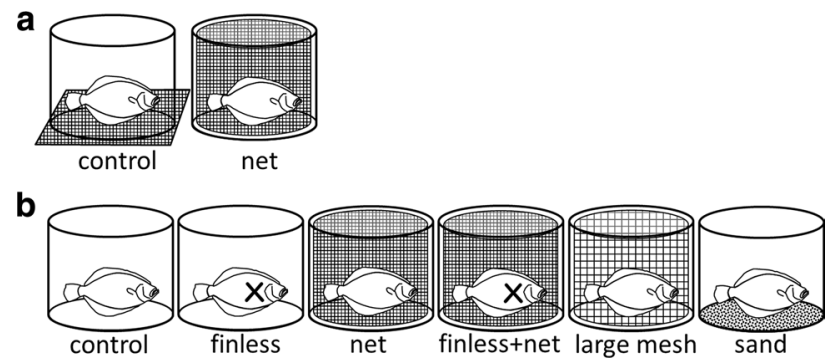

Fig. 1 Schematic illustrations of the experimental set-ups. a Experiment 1: effect of a net-lined rearing tank on juvenile Japanese flounder Paralichthys olivaceus before the appearance of hypermelanosis. b Experiment 2: hypermelanosis suppression in the axilla area. $X$ on the blind side indicates pectoral fin ablation. Control, intact juveniles in a tank without a net lining, finless pectoral fin-ablated juveniles in a tank without a net lining, net intact juveniles in a tank with a standard net lining, finless +net pectoral fin-ablated juveniles in a tank with a standard net lining, large mesh intact juveniles in a tank with a net lining made from netting with a larger mesh size, sand intact juveniles in a tank with a 1-cm-deep sandy bottom

tanks with a light-blue plastic sheet (sky blue, SK Event Sheet; Tanaka Sanjiro, Fukuoka, Japan).

At the start of the experiment (19 December 2016), juveniles were randomly selected from the stock tank, and 70 were assigned to each experimental tank. The remaining 73 juveniles were anaesthetized with $0.02 \%$ 2-phenoxyethanol, and their blind sides were photographed with a digital camera (EOS Kiss Digital DS5041; EFS 18-55 mm lens; both Canon, Tokyo, Japan). For all photographs, a scale was placed near the fish, and all the length of each fish and the darkened areas were calculated using the scale as standard. The standard length (SL) of the initial sample was $60 \pm 4 \mathrm{~mm}$ (mean \pm standard deviation [SD]), and 69 of the 73 individuals displayed no darkened areas on their blind sides. The total-darkened-area ratio (see section Measurement and statistical analysis for definition, similar to the ratio of darkening in Isojima et al. 2013a) for the four individuals with darkened areas was $12.6,1.6,1.5$, and $0.7 \%$, respectively.

The experimental tanks were set up indoors, and day length was controlled by ceiling fluorescent lights that were turned on at $0900 \mathrm{~h}$ and turned off at $1700 \mathrm{~h}$. Sand-filtered (natural sand $0.612 \mathrm{~mm}$ and anthracite $1.272 \mathrm{~mm}$ ), ultraviolet-sterilized (UV2500M-B; Ebara Corp., Tokyo, Japan) natural seawater was supplied at a rate of two rotations per day. Inlet water was supplied from a single tube placed just below the water surface near the tank wall, and outlet water was discharged by overflow through a filter placed near the tank wall. Both tanks were equally aerated using an air stone for each tank placed at the center of the tank. An ordinary siphon tub was used for daily cleaning of the tank bottom. Because excrement and remnant food were visible and only accumulated on a few places on the bottom, it took about
5 min to clean both tanks. The lowest and highest water temperatures during the experiment were $9.1{ }^{\circ} \mathrm{C}(10$ February 2017) and $18.0^{\circ} \mathrm{C}$ (16 April 2017), respectively. Commercial pellets (floating type, Hirame EPF-1 and EPF-3; Nissin Marubeni Feed Co., Ltd., Tokyo, Japan) were provided in the morning and afternoon. At the end of experiment (after 122 days, 20 April 2017), all of the juveniles from both tanks were photographed, as described in preceding paragraph.

\section{Experiment 2: Hypermelanosis suppression in the axilla area}

Darkening around the axilla area was not suppressed by the net-lined rearing tank in experiment 1 , probably due to the interruption of contact between the net and the skin caused by the pectoral fin of the blind side. We therefore attempted to increase this contact by ablation of the pectoral fin and the use of a larger mesh size.

This experiment was carried out at Maizuru Fisheries Research Station, Kyoto University, using juvenile flounders produced at, and kindly provided by, the Futtsu Laboratory, Institute of Seed Production, Chiba Prefectural Fishery Research Center. Fertilized eggs were obtained from wildcollected adults and reared following the standard protocol of the center. On 25 and 26 May 2017 (69 and 70 days, respectively, after hatching), juveniles of approximately $60 \mathrm{~mm}$ SL with a small darkened area on their blind side were selected for the experiment. Based on the results of our previous study, we assumed that similarly sized Japanese flounders produced at the Futtsu Laboratory which displayed a small darkened patch would, if left untreated, express significant hypermelanosis after 2 months (Isojima et al. 2013a). These fish were therefore suitable candidates for experiments on the suppression of hypermelanosis.

The juveniles were shipped to the Maizuru Fisheries Research Station on 31 May 2017 and arrived at this destination on 1 June 2017. Until the start of experiment (8 June 2017), all juveniles were reared in a 500-1 circular tank with a sandy bottom (thickness of sand layer $1 \mathrm{~cm}$, Micros ceramic, MS-1; NORRA Co., Ltd., Ootsu, Japan). During the acclimation period, the juveniles were treated with antibiotics (ERUBAJYU for aquaculture, $50 \mathrm{ppm}$; Ueno Pharmaceutical Co., Ltd., Osaka, Japan).

Six experimental tanks (transparent 200-1 circular tanks, polycarbonate; Tanaka Sanjiro, Fukuoka, Japan) were set up as treatments (Fig. 1b): 1 (control), intact juveniles in a tank without a net lining; 2 (finless), pectoral fin-ablated juveniles in a tank without a net lining; 3 (net), intact juveniles in a tank with a standard net lining; 4 (finless + net), pectoral fin-ablated juveniles in a tank with a standard net lining; 5 (large mesh), intact juveniles in a tank with a net lining made from netting with a larger 
mesh size; 6 (sand), intact juveniles in a tank with a sandy bottom (Micros ceramic, MS-1) that was about $1 \mathrm{~cm}$ deep.

Ablation of the pectoral fin on the blind side was performed with dissection scissors on fish under anesthesia using $0.01 \%$ 2-phenoxyethanol. When the length of the regenerated pectoral fin exceeded the mesh size $(4 \mathrm{~mm})$, the fin was again ablated on the 21 st day. Thereafter, the length of the regenerated pectoral fin did not exceed $4 \mathrm{~mm}$.

For the net-lined treatments (3: net) and (4: finless + net), linings similar to those used in experiment 1 were prepared using the same netting after the size had been adjusted to fit the 200-1 tank. For the net-lined treatment with the larger mesh size (5: large mesh), a mesh size larger than the length of pectoral fin of blind side was used. This allowed the fin to protrude more easily through the mesh, giving the axilla area of the blind side a higher probability of making contact with the net. To estimate the pectoral fin length from the total length of the juveniles, the relationship between the total length and the length of the pectoral fin of the blindside was determined based on preserved specimens of juvenile flounders; this was expressed as the following equation:

(pectoral fin length, $\mathrm{mm})=0.0815 \times($ total length, $\mathrm{mm})$

$$
+1.1848\left(N=45, R^{2}=0.9701\right) \text {. }
$$

Consequently, taking growth of the juveniles into consideration, we used a lining made from $12-\mathrm{mm}$ mesh netting (AIDEA net, Raschel knitted, polyethylene, green; Dio Chemical Ltd.) for the first 7 weeks of rearing, after which 25-mm mesh netting was used.

At the beginning of the experiment (8 June 2017), juveniles without hypermelanosis on the axilla area were selected. Among these, 19 juveniles were randomly selected as the initial sample, anesthetized with $0.01 \%$ 2-phenoxyethanol, and their blind sides photographed with a digital camera (STYLUS TG-630 Tough; Olympus, Tokyo, Japan). For all photographs, a scale was placed near the fish, and the length of each fish and the darkened areas were calculated using the scale as standard. The SL of the initial sample was $62 \pm 5 \mathrm{~mm}($ mean $\pm \mathrm{SD})$. The median, 25 percentile, and 75 percentile of the totaldarkened-area ratio were $1.15,0.74$, and $1.54 \%$, respectively. Nineteen juveniles were randomly assigned to each experimental tank.

The bottom and vertical walls of the tanks were covered from the outside with light-blue plastic sheets, as in experiment 1 . Sand-filtered $(0.6 \mathrm{~mm})$ and aerated natural seawater was supplied at a rate of 10 rotations per day. Inlet water was supplied from a single tube placed just above the center of water surface, and outlet water was discharged by overflow through a filter placed at the center of the tank. All tanks were equally aerated using an air stone for each tank placed at the center of the tank. An ordinary siphon tub was used for daily cleaning of the bottom of the flat-bottom tanks (control and finless) and net-lined tanks (net, finless + net, and large mesh). Because excrement and remnant food was visible and only accumulated on a few places on the bottom, it took less than about 2 min to clean the flat-bottom tanks and less than about $3 \mathrm{~min}$ to clean the net-lined tanks. However, daily cleaning of the bottom of the sand tank took $>15 \mathrm{~min}$ because we had to take out most of the sand and wash it stringently to get it clean. During the experiment, the water temperature was gradually increased from $19^{\circ} \mathrm{C}$ (at the start of experiment, 8 June 2017) to $27^{\circ} \mathrm{C}$ (at the end, 4 August 2017). The tanks were set up indoors without exposure to direct sunlight, and the day length was controlled by ceiling fluorescent lights that were turned on at $0600 \mathrm{~h}$ and turned off at $1800 \mathrm{~h}$. Commercial pellets (sinking type, Otohime S2 and EP2; Nissin Marubeni Feed Co.) were provided in the morning and afternoon.

Final sampling was performed 57 days after the start of rearing (4 August 2017). All juveniles were euthanized with $0.1 \%$ 2-phenoxyethanol and photographed as in section on experiment 1.

\section{Collection of wild-caught juveniles}

The body proportions of wild-caught Japanese flounder juveniles were measured for comparison. The wild-caught juveniles were caught at depths of 5-10 m in western Wakasa Bay, Kyoto Prefecture, on 13 July 2016. The specimens were preserved in $99 \%$ ethanol until measurement.

\section{Measurement and statistical analysis}

In this experiment, all lengths and areas were measured from digital images using ImageJ (National Institute of Health, Bethesda, MD, USA; https://imagej.nih.gov/ij/). Because photographs were taken using "auto mode", pigmented areas were manually identified on the display. The total-darkened-area ratio was defined as the ratio of total pigmented areas to the entire blind side area without the fins. For more detail, the hypermelanosis was classified into the following four areas on the blind side (Fig. 2): along the base of the dorsal and anal fins (marginal area, Fig. 2a), around the base of the pectoral fin (axilla area, Fig. 2b), around the base of the pelvic fin (abdominal area, Fig. 2c), and the center of the head (head area, Fig. 2d). The axilla-darkened-area ratio was defined as the ratio of the pigmented area in the axilla region to the entire blind side area without fins. For body depth, the largest body depth along the dorso-ventral axis without fins was measured. 


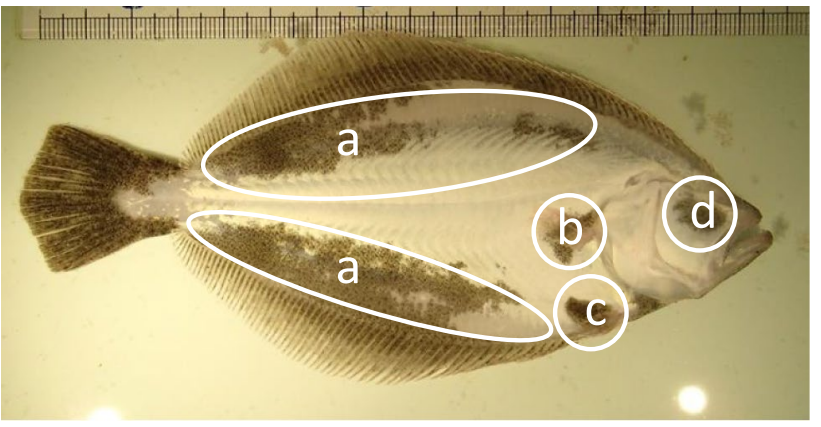

Fig. 2 Typical darkened areas on the blind side of Japanese flounder. Hypermelanosis typically emerges in four areas independently on the blind side: the marginal area $(a)$, the axilla area $(b)$, the abdominal area $(c)$, and the head area $(d)$. Other darkened areas (near the caudal fin, for example) were also measured and included in total darkened area, but the occurrence of such areas was not counted separately in Table 1

For data values whose normality and equality of variance were not rejected by the Shapiro-Wilks test and Bartlett test, respectively, the statistical differences among the averages were parametrically examined by Student $t$ tests (two groups). Parametrical tests for three groups or more were not needed in the study. For other values, statistical analyses were non-parametrically examined by the Mann-Whitney $U$ test (two groups) or the Kruskal-Wallis test (three groups or more), followed by the Steel-Dwass post hoc test. For frequencies, statistical significance was tested by Fisher's exact test. The significance level was set at 0.05. All statistical analyses were performed using EZR (Kanda 2013; available at https://www.jichi.ac.jp/saitamasct/, Saitama Medical Center, Jichi Medical University, Saitama, Japan), which is a graphical user interface for $\mathrm{R}$ (R Core Team 2014).

\section{Results}

\section{Experiment 1: Effect of a net-lined rearing tank on juveniles before the appearance of hypermelanosis}

Figure 3 shows the blind side photographs of 24 juveniles randomly selected from each tank. Juveniles with large darkened areas were abundant in the control tank, but very rare in the net-lined tank. The total-darkened-area ratio was significantly lower in the juveniles in the net-lined tank than in those in the control tank (Fig. 4; Mann-Whitney $U$ test, $p<0.05)$. The median total-darkened-area ratio of juveniles in the net-lined tank $(0.5 \%)$ was about $1 / 40$ th of that of juveniles in the control tank $(20 \%)$.

Table 1 shows the number of individuals in each tank that developed darkened skin areas in each blind side area and

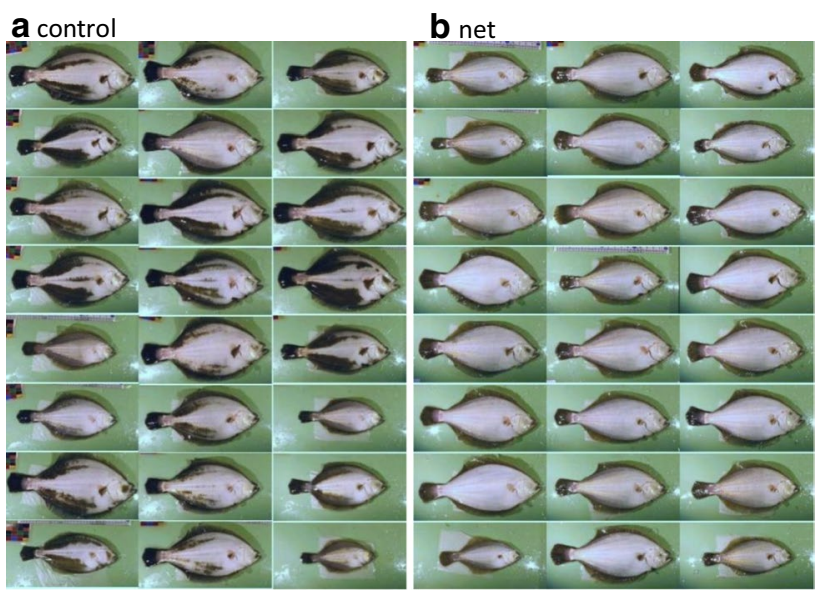

Fig. 3 Photographs of the blind side of juvenile Japanese flounder reared in the control tank (a, control) and net-lined tank (b, net) in experiment 1 . The blind sides of 24 randomly selected juveniles are shown

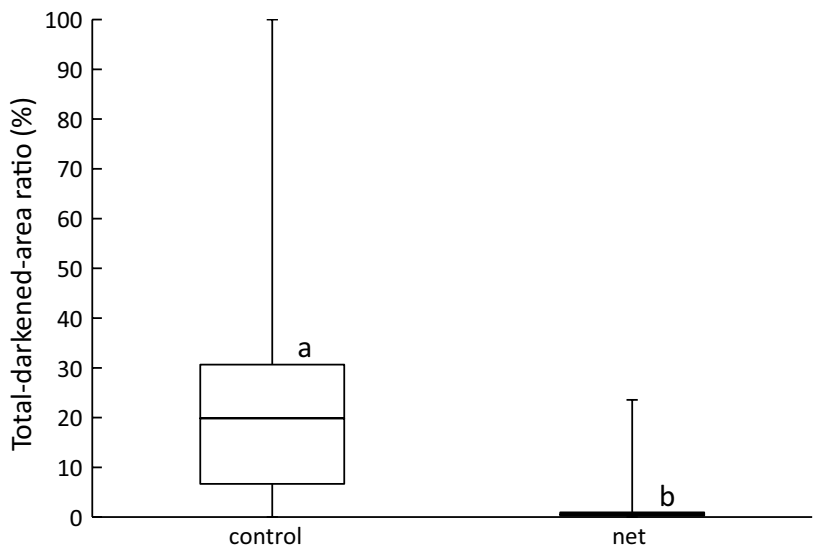

Fig. 4 Boxplot of total-darkened-area ratio of juvenile Japanese flounder reared in the control and net-lined (net) tanks in experiment 1. Different lowercase letters indicate statistical significance between groups (Mann-Whitney $U$ test, $p<0.05$ ). The upper and lower ends of the vertical bars show the maximum and minimum values, respectively. The top and bottom of the boxes show the 75 and $25 \%$ values, respectively, and the horizontal line in the box shows the median value

the total number of individuals with hypermelanosis at the end of the experiment. The appearance of darkened areas was significantly suppressed in the marginal, abdominal, and head areas of juveniles in the net-lined tank (Fisher exact test, $p<0.05$ ), but not in the axilla area (Fisher exact test, $p>0.05)$. There was no statistical difference in survival rate between juveniles in the control and net-lined tanks (Fisher exact test, $p>0.05$ ).

At the end of experiment 1, the median SL of the fish in the net-lined tank was $154.8 \mathrm{~mm}$, which was significantly smaller (by approx. 5\%) than that of the fish in the control 
Table 1 Number of individuals of Japanese flounder Paralichthys olivaceus with darkened skin at various areas of the blind side in experiment 1

\begin{tabular}{llllll}
\hline Group & Marginal & Axilla & Abdominal & Head & (Total) \\
\hline Control & $66 \mathrm{a}$ & $58 \mathrm{a}$ & $52 \mathrm{a}$ & $40 \mathrm{a}$ & 66 \\
Net & $1 \mathrm{~b}$ & $57 \mathrm{a}$ & $28 \mathrm{~b}$ & $14 \mathrm{~b}$ & 68 \\
\hline
\end{tabular}

Different lowercase letters indicate statistical significance between groups (Fisher exact test, $p<0.05$ )

tank (163.4 mm) (Fig. 5; Mann-Whitney $U$ test, $p<0.05)$. In addition, the ratio of body depth to SL (body depth:SL ratio) was slightly, but significantly, smaller in juveniles reared in the net-lined tank $(0.42 \pm 0.02$, mean $\pm S D)$ than in those reared in the control tank $(0.04 \pm 0.02)(t$ test, $p<0.05)$.

\section{Experiment 2: Hypermelanosis suppression in the axilla area}

The total-darkened-area ratios at the end of experiment 2 are shown in Fig. 6. Regardless of the presence or absence of a net lining, ablation of the pectoral fin had no significant effect on the total-darkened-area ratio (Steel-Dwass test, $p>0.05$ ). Furthermore, regardless of the presence or absence of the pectoral fin, experimental groups in tanks without a net lining showed significantly higher values for the total-darkened-area ratio than did those in tanks with net linings or sand (Steel-Dwass test, $p<0.05$ ). The totaldarkened-area ratio of the group in the tank with a sandy bottom group was significantly lower than that of all other experimental groups (Steel-Dwass test, $p<0.05$ ).

At the end of experiment 2, only one juvenile had died, in the large mesh treatment, and there was no statistical

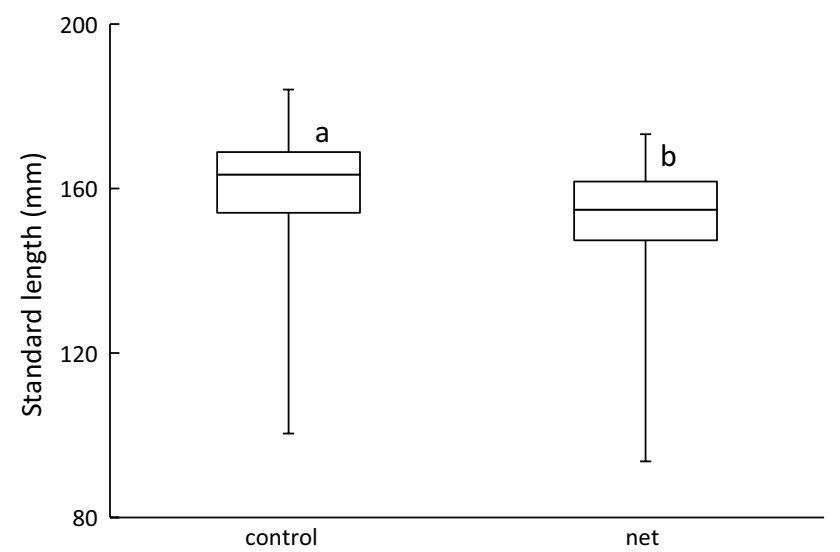

Fig. 5 Boxplot of standard length of juvenile Japanese flounder reared in the control and net-lined (net) tanks in experiment 1 . The upper and lower ends of the vertical bars show the maximum and minimum values, respectively. The top and bottom of the boxes show the 75 and $25 \%$ values, respectively, and the horizontal line in the box shows the median value

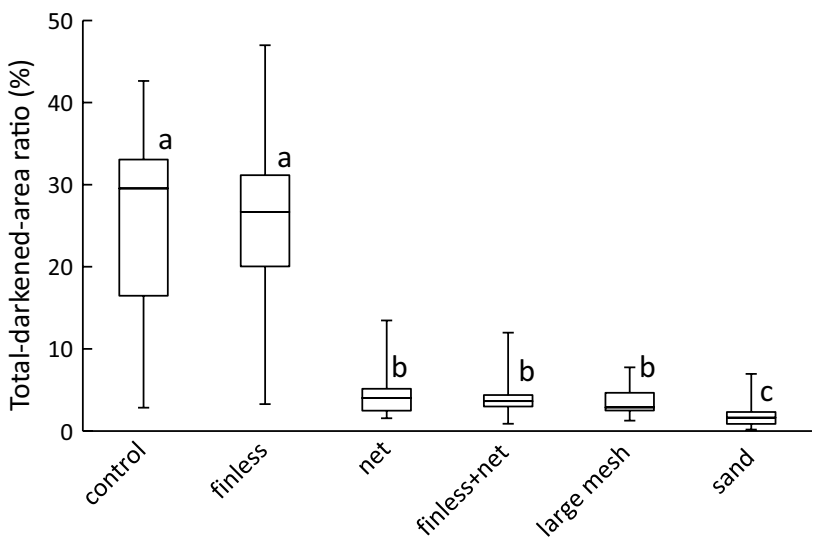

Fig. 6 Boxplot of total-darkened-area ratio in juvenile Japanese flounder in experiment 2. Different lowercase letters indicate statistical significance between groups (Steel-Dwass test, $p<0.05$ ). There were 18-19 individuals in each group. The upper and lower ends of the vertical bars show the maximum and minimum values, respectively. The top and bottom of the boxes show the 75 and $25 \%$ values, respectively, and the horizontal line in the box shows the median value

difference in the survival rate among treatments (Fisher's exact test, $p>0.05)$. The numbers of individuals with darkened axilla areas were 19/19 (control), 19/19 (finless), 19/19 (net), 18/19 (finless + net), 18/18 (large mesh), and $17 / 19$ (sand), respectively; there was no statistical difference among the treatments (Fisher's exact test, $p>0.05$ ). The results clearly show that the initial appearance of the darkened areas around the axilla was not suppressed by any treatment.

The axilla-darkened-area ratios are shown in Fig. 7. Regardless of the presence or absence of a net, the absence of the pectoral fin tended to suppress the size of the darkened areas around the axilla; however, the differences were not significant (Steel-Dwass test, $p>0.05$ ). In addition, compared to juveniles in the control tank, the axilla-darkened-area ratio was significantly lower in juveniles in the finless + net, large mesh, and sand tanks (Steel-Dwass test, $p<0.05$ ).

The SL and body depth:SL ratio values at the end of experiment 2 are shown in Fig. 8. The SL values were significantly larger in juveniles in tanks without net linings (SL $140 \mathrm{~mm}$; control, finless, and sand treatments) than in those with net linings (SL approx. $117 \mathrm{~mm}$; net, finless + net, and large mesh treatments) (Steel-Dwass test, $p<0.05)$. Although the body size of wild-caught Japanese flounder juveniles was much smaller (SL 69 mm; Fig. 8a), we attempted a preliminary comparison between the body proportions of the experimental and wild-caught juveniles (Fig. 8b). The body depth:SL ratios of the wild-caught juveniles were more similar to those from tanks with net linings (about 0.39) than they were to those from tanks without net linings (about 0.42). 


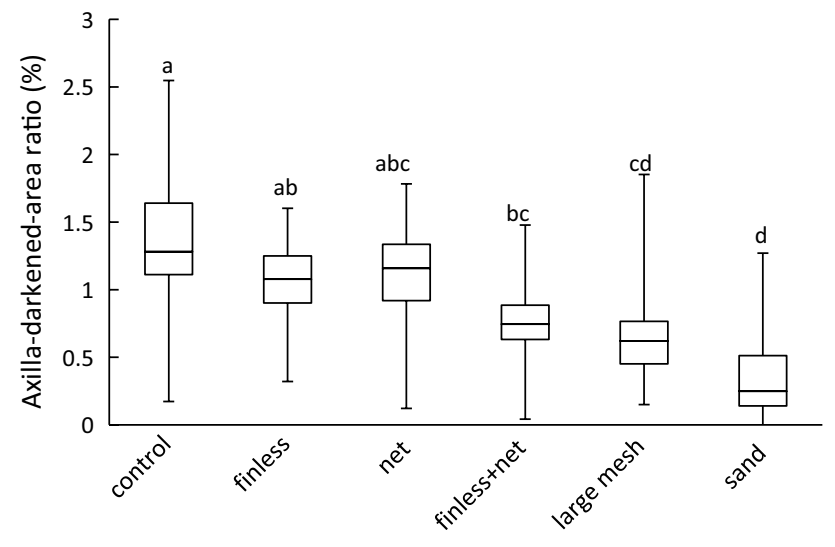

Fig. 7 Boxplot of axilla-darkened-area ratio in juvenile Japanese flounder in experiment 2. Different lowercase letters indicate statistical significance between groups (Steel-Dwass test, $p<0.05$ ). There were 18-19 in each group. The upper and lower ends of the vertical bar show the maximum and minimum values, respectively. The top and bottom of the boxes show the 75 and $25 \%$ values, respectively, and the horizontal line in the box shows the median value

\section{Discussion}

In this study, we first showed that net-lined rearing tanks clearly suppress hypermelanosis in Japanese flounders, both it its expansion in juveniles with small darkened areas and also its significant initial appearance in juveniles without darkened areas. Darkening in the axilla area, the most difficult area in which to suppress hypermelanosis, could also be suppressed by selecting netting with a larger mesh size. However, the juveniles reared in net-lined tanks experienced slower growth and had different body proportions to those of traditionally reared juvenile.

\section{Effect of net-lined rearing tanks on hypermelanosis and growth in juvenile flounders}

Nakata et al. (2017) demonstrated that an undulating bottom surface, such as that provided by a net lining, suppressed the "expansion" of hypermelanosis in juveniles that had already expressed a small degree of darkening. To examine whether net-lined rearing tanks also prevent the initial appearance of hypermelanosis, the juveniles were subjected to the treatments mostly before the appearance of any darkened areas. The initial appearance of hypermelanosis, especially in the marginal area, was almost entirely prevented (Table 1) and, consequently, the total darkened area was remarkably reduced to about $1 / 40$ th of that of traditionally reared juveniles (Figs. 3, 4). This clearly indicates the effectiveness of the net lining in suppressing the initial appearance of hypermelanosis. In terms of hypermelanosis expansion, the results of experiment 2 are basically consistent with those Nakata et al. (2017), in which juveniles reared in a net-lined tank tended to show smaller areas of hypermelanosis than those reared in flat-bottom tank of the same bottom color. The total-darkened-area ratios were significantly smaller in all three tanks with net linings than they were in the two tanks without net linings (Fig. 6). Only a single tank was used for each treatment in the present study due to the limited equipment available, but the results were consistent between the tanks with and without net linings, indicating the strong reproducibility of the hypermelanosis-suppressing effect of net-lined rearing tanks.

Although rearing juveniles in sandy-bottomed tanks strongly prevents hypermelanosis (Seikai 1991; Iwata and Kikuchi 1998; Kang and Kim 2012, 2013c; Isojima et al. 2013b, 2014), a sandy bottom requires more intense tank

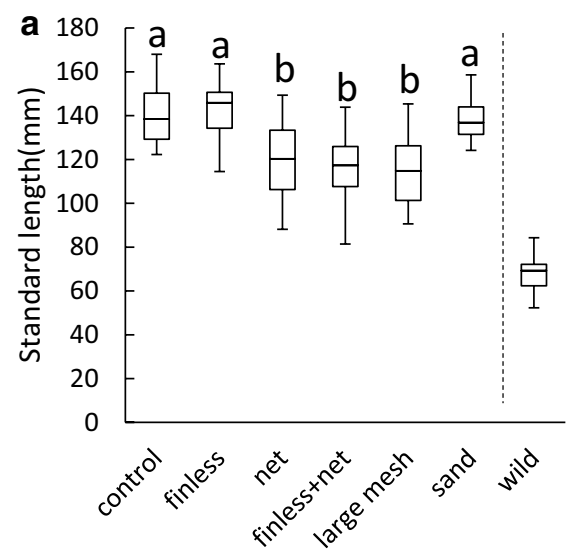

Fig. 8 Boxplot of standard length (SL) and body proportion in juvenile Japanese flounder in experiment 2. a SL, b body depth:SL ratio. The number of individuals was 18-19. Values obtained from 15 wild-caught juveniles were added to the graphs, but not included in the statistical test. Different lowercase letters indicate statistical sig-

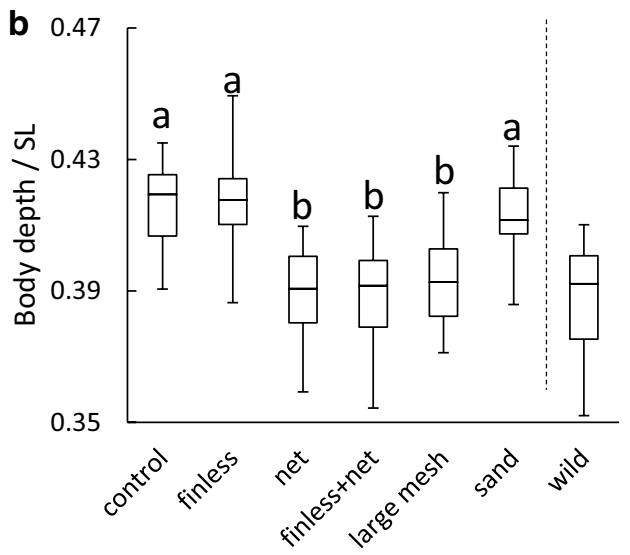

nificance between groups (Steel-Dwass test, $p<0.05$ ). The upper and lower ends of the vertical bars show the maximum and minimum values, respectively. The top and bottom of the boxes show the 75 and $25 \%$ values, respectively, and the horizontal line in the box shows the median value 
cleaning. A net lining can be an excellent substitute for bottom sand and is a practical method for preventing hypermelanosis; however, this approach needs to be confirmed in industrial-scale tanks holding a much larger number of juveniles.

This study also revealed two drawbacks to net-lined rearing tanks. The first is slower growth. Nakata et al. (2017) also reported lower growth rates in juveniles reared in netlined tanks. In that study, the juveniles were provided with a diet of sinking-type pellets, and a significant proportion these became inaccessible when they fell through the mesh and remained under the net. The authors thus speculated that the lower growth rate of the fish reared in the net-lined tanks was caused by a lower amount of nutrients being available compared to the amount available to fish reared in the flat-bottom tanks (control tank). This situation was identical to that in experiment 2 of the present study. In experiment 1 , although a diet of floating-type pellets was provided, a significant number of these pellets still escaped consumption and fell through the net where they were inaccessible to the fish. It is possible that the lower growth rate was again caused by fewer nutrients being available to fish reared in the net-lined tanks. Thus, before these net-lined rearing tanks can be applied at an industrial scale, methods of keeping the pellets inside the net are required to enable growth equivalent to that of traditionally reared flounders.

\section{Prevention of axilla darkening}

Another drawback of the net-lined rearing tanks is their ineffectiveness in suppressing axilla darkening. As shown in Table 1, the net-lined rearing tank significantly reduced the appearance of darkening in the marginal, abdominal, and head areas. However, although the axilla area is much smaller than the marginal area, the appearance of darkening in this area was not suppressed, possibly due to the presence of the pectoral fin between the blind side skin of the axilla area and the net. If contact with the bottom substrate suppresses darkening (Nakata et al. 2017), then the presence of a pectoral fin could interfere with this contact with the net, resulting in the appearance of darkening in this specific area. Therefore, we examined the effect of pectoral fin ablation.

The results of experiment 2 show that the ablation of the pectoral fin did not affect the SL (Fig. 8a) or the total-darkened-area ratio (Fig. 6), regardless of rearing in the presence or absence of a net lining. These results suggest that fin ablation does not cause any major defects affecting growth. It has been speculated that fin ablation does not induce serious chronic stress because stress-induced cortisol is known to increase hypermelanosis (Matsuda et al. 2018b).

Although the initial appearance of darkening in the axilla area was not suppressed by any of the treatments in experiment 2 , the expansion of darkened areas did tend to be suppressed by pectoral fin ablation (Fig. 7). Therefore, the presence of the pectoral fin is a possible reason for the expansion of darkening in the axilla area.

The use of the net linings with larger mesh sizes resulted in a significantly smaller area of darkening in the axilla region compared to the control; also, the smaller area of darkening in the axilla region of juveniles reared in net-lined tanks was comparable to the area in juveniles raised in the sandy bottom tank (Fig. 7). We selected a mesh size larger than the pectoral fin of the blind side because we expected that the protrusion of the pectoral fin through the mesh would provide better contact between the net and the skin of the axilla area. The resulting suppression of hypermelanosis further supports the notion that the presence of a pectoral fin induces local darkening in the axilla area by interfering with the hypermelanosis-suppressing effect of contact stimulation from the bottom substrate (Nakata et al. 2017). Net-lined rearing tanks using nets with larger mesh sizes can therefore be an excellent solution for preventing hypermelanosis.

\section{Differences in body proportion}

Wild-caught juveniles with a SL of $40-110 \mathrm{~mm}$ are known to have a constant body depth:SL ratio of approximately 0.38 (Yoshimura and Kawashita 2003), which is smaller than that of artificially raised juveniles (Nihira 1990; Yoshimura and Kawashita 2003). In the present study, the body depth:SL ratio was significantly lower in juveniles reared in tanks with net linings, with the ratios in experiment 2 (Fig. 8b) being similar to that of wild-caught juveniles (0.39) of a smaller size. Similar to the juveniles reared in the net-lined tanks, but inconsistent to those reared in the sandy bottom tank of the present study, juveniles reared in tanks with bottom sand or a sand-pasted bottom in the study of Kawana and Namba (2004) displayed significantly smaller body depth:SL ratios (0.37-0.38) than those reared in control tank (0.39-0.40). In addition, the present study showed differing values between experiment 1 and experiment 2, with the body depth:SL ratios of the control groups being 0.44 and 0.42 , respectively, and those of the net groups being 0.42 and 0.39 , respectively; this trend was roughly similar to that of the SL of each group. Although contact stimulation from the bottom substrate is possibly involved, growth-dependent changes in body shape cannot be excluded as a reason for the difference. Further study is required to elucidate this point.

\section{Optimal application of the net-lining method}

In this study, better results in terms of less hypermelanosis in the axilla area were achieved using the larger mesh size $(12 \mathrm{~mm})$, while the results of total hypermelanosis suppression were comparable between the 12- and 4-mm mesh treatments. Theoretically, a larger mesh should be less 
effective due to less skin contact with the net; the contact frequency of a given skin area is expected to be proportional to the length of the strands constituting each unit area of the net. For example, when a net with a mesh size of $12 \mathrm{~mm}$ is used, the frequency of contact with the skin is expected to decrease to one-third of that when the net used has a mesh size of $4 \mathrm{~mm}$. It is also possible that a smaller mesh size provides a smoother touch and, therefore, less stimulation to the skin. In addition, the mesh size of the net lining must be small enough not to let the juveniles through, and large enough to allow excrement through. It is still unknown whether different mesh sizes would be optimal for differently sized juveniles or whether a single optimal mesh size applies for all fish size classes. For factors other than mesh size, it also needs to be examined whether other types of nets (for example, knitting methods other than Raschel, and materials other than polyethylene) also prevent hypermelanosis. If we are able to answer these questions and if we improve the installation methods for net linings in larger tanks, then the utilization of nets as a bottom substrate may become an excellent solution for the prevention of hypermelanosis in flatfish aquaculture.

Acknowledgements We would like to thank Ootawa Shubyo, Saikai, Nagasaki, and Futtsu Laboratory, Institute of Seed Production, Chiba Prefectural Fisheries Research Center, for kindly providing juvenile Japanese flounder. We would also like to thank the members of the Laboratory of Marine Stock-Enhancement Biology and Maizuru Fisheries Research Station, Kyoto University, for their invaluable discussions and encouragement throughout the course of the study. This work was supported by JSPS KAKENHI Grant number 15K07575 to M.T.

Open Access This article is licensed under a Creative Commons Attribution 4.0 International License, which permits use, sharing, adaptation, distribution and reproduction in any medium or format, as long as you give appropriate credit to the original author(s) and the source, provide a link to the Creative Commons licence, and indicate if changes were made. The images or other third party material in this article are included in the article's Creative Commons licence, unless indicated otherwise in a credit line to the material. If material is not included in the article's Creative Commons licence and your intended use is not permitted by statutory regulation or exceeds the permitted use, you will need to obtain permission directly from the copyright holder. To view a copy of this licence, visit http://creativecommons.org/licenses/by/4.0/.

\section{References}

Amiya N, Amano M, Takahashi A, Yamanome T, Kawauchi H, Yamamori K (2005) Effects of tank color on melanin-concentrating hormone levels in the brain, pituitary gland, and plasma of the barfin flounder as revealed by a newly developed time-resolved fluoroimmunoassay. Gen Comp Endocrinol 143:251-256

Haga Y, Takeuchi T, Murayama Y, Ohta K, Fukunaga T (2004) Vitamin D3 compounds induce hypermelanosis on the blind side and vertebral deformity in juvenile Japanese flounder Paralichthys olivaceus. Fish Sci 70:59-67

Isojima T, Makino N, Takakusagi M, Tagawa M (2013a) Progression of staining-type hypermelanosis on the blind side in normally metamorphosed juveniles and pigmentation progression in pseudoalbino juveniles of the Japanese flounder Paralichthys olivaceus using individual identification. Fish Sci 79:787-797

Isojima T, Tsuji H, Masuda R, Tagawa M (2013b) Formation process of staining-type hypermelanosis in Japanese flounder juveniles revealed by examination of chromatophores and scales. Fish Sci 79:231-242

Isojima T, Makino N, Miyama Y, Tagawa M (2014) Effects of time and duration of rearing with bottom sand on the occurrence and expansion of staining-type hypermelanosis in the Japanese flounder Paralichthys olivaceus. Fish Sci 80:785-794

Iwata N, Kikuchi K (1998) Effects of sandy substrate and light on hypermelanosis of the blind side in cultured Japanese flounder Paralichthys olivaceus. Environ Biol Fish 52:291-297

Kaji S, Fukunaga T (1999) Results of a questionnaire on the recent status of seed production and market price of recaptured Japanese flounder Paralichthys olivaceus showing abnormal coloration. Saibai Giken 27:67-101 (in Japanese)

Kanda Y (2013) Investigation of the freely available easy-to-use software "EZR" for medical statistics. Bone Marrow Transplant 48:452-458

Kang DY, Kim HC (2012) Relevance of environmental factors and physiological pigment hormones to blind-side hypermelanosis in the cultured flounder, Paralichthys olivaceus. Aquaculture 356-357:14-21

Kang DY, Kim HC (2013a) Functional characterization of two melanin-concentrating hormone genes in the color camouflage, hypermelanosis, and appetite of starry flounder. Gen Comp Endocrinol 189:74-83

Kang DY, Kim HC (2013b) Influence of density and background color to stress response, appetite, growth, and blind-side hypermelanosis of flounder, Paralichthys olivaceus. Fish Physiol Biochem 39:221-232

Kang DY, Kim HC (2013c) Importance of bottom type and background color for growth and blind-side hypermelanosis of the olive flounder, Paralichthys olivaceus. Aquac Eng 57:1-8

Kang DY, Kim HC (2015) Functional relevance of three proopiomelanocortin (POMC) genes in darkening camouflage, blindside hypermelanosis, and appetite of Paralichthys olivaceus. Comp Biochem Physiol B 179:44-56

Kawana K, Namba K (2004) Suppression effect of contact stimuli on abnormal body coloration of blind side in Japanese flounder. In: Fukunaga T, Shiozawa S, Tsuzaki T (eds) Induction factors and prevention methods for body color abnormality on the blind side of Japanese flounder. Stock-enhancement technology series 10. Fisheries Research Agency, Tokyo, pp 127-134 (in Japanese)

Matsuda N, Kasagi S, Nakamaru T, Masuda R, Takahashi A, Tagawa M (2005) Left-right pigmentation pattern of Japanese flounder corresponds to expression levels of melanocortin receptors (MC1R and MC5R), but not to agouti signaling protein 1 (ASIP1) expression. Gen Comp Endocrinol 262:90-98

Matsuda N, Yamamoto I, Masuda R, Tagawa M (2018) Cortisol promotes staining-type hypermelanosis in juvenile Japanese flounder. Aquaculture 497:147-154

Nakamura M, Seikai T, Aritaki M, Masuda R, Tanaka M, Tagawa M (2010) Dual appearance of xanthophores, and ontogenetic changes in other pigment cells during early development of Japanese flounder Paralichthys olivaceus. Fish Sci 76:243-250

Nakata K, Yamamoto I, Miyama Y, Nakamaru T, Masuda R, Tagawa M (2017) Undulated flooring in the rearing tank decreases hypermelanosis in Japanese flounder Paralichthys olivaceus. Fish Sci 83:1027-1035

Nihira A (1990) Difference between ratio of body depth to total length of wild flounders and artificial ones. Bull Fish Exp St Ibaraki-Ken 28:113-115 (in Japanese) 
R Core Team (2014) R: a language and environment for statistical computing. R Foundation for Statistical Computing, Vienna, Austria. https://www.R-project.org/. Accessed June 2017

Seikai T (1999) nfluences of fluorescent light irradiation, ocular side pigmentation, and source of fishes on the blind side pigmentation in the young Japanese flounder, Paralichthys olivaceus. Aquac Sci 39:173-180 (in Japanese with English abstract)

Seikai T, Matsumoto J, Shinozaki M, Oikawa A, Akiyama T (1987) An association of melanophores appearing at metamorphosis as vehicles of asymmetric skin color formation with pigment anomalies developed under hatchery conditions in the Japanese flounder, Paralichthys olivaceus. Pigment Cell Res 1:143-151

Tarui F, Haga Y, Ohta K, Shima Y, Takeuchi T (2006) Effect of Artemia nauplii enriched with vitamin A palmitate on hypermelanosis on the blind side in juvenile Japanese flounder Paralichthys olivaceus. Fish Sci 72:256-262

Yamanome T, Amano M, Amiya N, Takahashi A (2007) Hypermelanosis on the blind side of Japanese flounder Paralichthys olivaceus is diminished by rearing in a white tank. Fish Sci 73:466-468
Yamanome T, Amano M, Takahashi A (2005) White background reduces the occurrence of staining, activates melanin-concentrating hormone and promotes somatic growth in barfin flounder. Aquaculture 244:323-329

Yamanome T, Chiba H, Takahashi A (2007) Melanocyte-stimulating hormone facilitates hypermelanosis on the non-eyed side of the barfin flounder, a pleuronectiform fish. Aquaculture 270:505-511

Yoshimura K, Kawashita M (2003) Comparison of body proportions and meristic characters between hatchery-reared and wild Japanese flounder juveniles. Sci Rep Hokkaido Fish Exp Stn 64:105111 (in Japanese with English abstract)

Publisher's Note Springer Nature remains neutral with regard to jurisdictional claims in published maps and institutional affiliations. 